\title{
Determinantes do desemprego recente no Rio Grande do Norte e na região metropolitana de Natal - 2001-2008
}

\author{
Luís Abel da Silva Filho* \\ Reili Amon-Há Vieira dos Santos** \\ Maria do Livramento Miranda Clementino ${ }^{* * *}$
}

\begin{abstract}
RESUMO - O desemprego no Brasil afetou acentuadamente a população nas últimas duas décadas do século XX e adentrou os anos iniciais do século XXI com forte resistência às políticas de combate a ele. Somente com a melhora observada nos indicadores macroeconômicos nacionais, a partir do final de 2003 e início de 2004, é que se percebeu melhora relativa nos indicadores de emprego no país. Diante disso, o mercado de trabalho tem se mostrado seletista e excludente para boa parcela da população. Destarte, é pretensão deste estudo observar o perfil do desemprego recente no estado do Rio Grande do Norte $(\mathrm{RN})$ e na região metropolitana de Natal (RMN). Os dados são da Pesquisa Nacional por Amostra de Domicílios (PNAD) e os anos selecionados compreendem o período entre 2001 e 2008. Os principais resultados evidenciam redução na taxa de desemprego na RMN e no estado do RN. Na primeira, a redução do desemprego foi constatada a partir de 2003 , sendo que no estado os efeitos do crescimento econômico do país só se apresentaram a partir de 2005 no mercado de trabalho. Além disso, perceberam-se maiores taxas de desemprego para mulheres, não brancos e jovens. Destacou-se ainda o desemprego mais elevado para a RMN do que para todo o estado em todos os anos observados. Tais evidências ratificam a hipótese de que o desemprego concentra-se, sobretudo, nas regiões metropolitanas do país.
\end{abstract}

Palavras-chave: Determinantes do desemprego. Rio Grande do Norte. Região metropolitana de Natal.

\section{INTRODUÇÃO}

Estudar o desemprego no Brasil dos anos 2000 requer sempre uma retomada à discussão do que foram os anos de 1990, e sua herança no cenário econômico brasileiro. Esses anos marcaram o mercado de trabalho nacional por diversos fatores que ocorreram e corroboraram nesse aspecto. Desde as conturbações no cenário político nacional até o insucesso das políticas de combate a inflação nos anos iniciais (POCHMANN, 1999) podem ser apontados como fatores que contribuíram com o desemprego e com a insegurança no mercado de trabalho * Mestre em Economia pela Universidade Federal do Rio Grande do Norte. É pesquisador do Observatório das Metrópoles da Universidade Federal do Rio Grande do Norte. Endereço eletrônico: abeleconomia@hotmail. com.

** Mestrando em Economia pela Universidade Federal do Rio Grande do Norte. Bolsista REUNI. Endereço eletrônico: reiliamon@gmail.com.

*** Doutora em Economia pela Universidade Estadual de Campinas. É professora titular do Departamento de Políticas Públicas da Universidade Federal do Rio Grande do Norte e coordenadora de rede do Observatório das Metrópoles, núcleo da Universidade Federal do Rio Grande do Norte. Endereço eletrônico: clement@ufrnet.br. 
brasileiro.

As elevadas taxas de desemprego registradas nos anos de 1990 (POCHMANN, 1999; BALTAR, 2003; ANTUNES, 2005) fazem parte de uma conjuntura macroeconômica que atingiu todos os países latino-americanos dentro de um contexto econômico de elevada dependência externa e de forte atuação do estado neoliberal, implementado aos moldes da propagação e domínio do liberalismo econômico, prioritariamente desenvolvido nos Estados Unidos.

A ideologia neoliberal, seguida da conjuntura política brasileira, norteada pelos problemas de gestão pública vivenciados no governo Collor, além de sua política de abertura econômica e de internacionalização da economia do país, marcaram profundamente o mercado de trabalho nacional nos anos de 1990. Essas ações perpassaram a década em questão e adentraram os anos 2000 com forte propagação do desemprego e da precarização das condições de trabalho.

Somente a partir de 2003, com registro de melhora nos indicadores macroeconômicos nacionais, dentre eles a elevação da taxa de crescimento do Produto Interno Bruto (DEDECCA; ROSANDISKI, 2003), é que se constatam os primeiros sinais de recuperação do contrato de trabalho com carteira assinada e da redução das taxas de desemprego aberto no Brasil (REMY; QUEIROZ; SILVA FILHO, 2010). Contudo, os resultados observados nos contratos de trabalho apontam para elevação destes com forte viés daqueles assistidos nos anos de 1990. Além disso, a discriminação e a seletividade no mercado de trabalho apresentam-se excessivamente acentuados, atingindo a maioria de jovens, idosos, não brancos e mulheres (HIRATA, 2009).

As áreas metropolitanas têm percebido tais constatações com mais veemência, dada a maior concentração populacional no território (CLEMENTINO et al., 2009a; CLEMENTINO et al., 2009b; CARVALHO, 2006) e a flexibilização da produção ter atingido mais as áreas de concentração de atividades produtivas, de sua forte migração para o interior dos estados em busca de incentivos fiscais (SILVA FILHO; QUEIROZ, 2009) e de maior liberdade de atuação, sem, contudo, perceber ação constante dos órgãos de defesa da classe trabalhista assalariada.

Destarte, é pretensão deste artigo, investigar as taxas e os determinantes do desemprego recente em todo o estado do Rio Grande do Norte, observando-se, também, a região metropolitana de Natal. Os dados são da Pesquisa Nacional por Amostra de Domicílios (PNAD) e compreendem os anos de 2001 até 2008.

Para atingir os objetivos propostos pelo estudo, o artigo está estruturado da forma que se segue: além desta introdução, na segunda seção, discute-se o desemprego recente no Brasil e suas causas; na terceira seção, recorre-se aos procedimentos metodológicos, onde se apresen- 
tam os dados da pesquisa e a construção dos indicadores que caracterizam o perfil do desemprego ora observado; na quarta seção, abordam-se os determinantes do desemprego, através da análise dos dados; na quinta seção, são feitas algumas considerações finais.

\section{CARACTERIZAÇÃO DO DESEMPREGO RECENTE NO BRASIL E NO CON- TEXTO METROPOLITANO}

As configurações econômicas mundiais e seus reflexos nas economias em desenvolvimento promoveram elevadas consequências no mercado de trabalho. A reestruturação produtiva e as configurações ocorridas na política brasileira proporcionaram um contexto de fortes ameaças aos assalariados do país. Assistiram-se a novas formas de contratação e a redução dos postos de trabalho dado, sobretudo, pelas modificações nas formas de produção e pela entronização da ideologia neoliberal dominante no final do século.

Em aspectos gerais, pode-se afirmar que o país vivenciou uma de suas maiores modificações no mercado de trabalho e registraram-se as maiores taxas de desemprego aberto já vivenciado no país. Além do desemprego aberto, o desemprego oculto pelo trabalho precário e pelo desalento castigou o mercado de trabalho brasileiro no final do século XX (BASTOS, 2010). Concomitantemente, assistiram-se a formas de contratos de trabalho por tempo parcial e por tempo indeterminado, registrando-se novas formas de contratos nunca vistos anteriormente no Brasil (NEVES; PEDROSA, 2007).

As regiões metropolitanas brasileiras foram acentuadamente afetadas, seja por concentrar contingente populacional elevado, seja por absorver, com maior intensidade, as inovações tecnológicas nas atividades produtivas que se infiltraram com a expansão da reestruturação produtiva. Somando-se a isso, destacam-se os parâmetros de internacionalização da economia brasileira, que se definiram com a abertura econômica no final do século passado.

Tais acontecimentos provocaram grandes consequências no mercado de trabalho brasileiro, sendo que, além do desemprego, viu-se a criação de postos de trabalho excessivamente precários com exclusão e seletividade acentuada na geração de emprego do país.

Os estudos acerca do desemprego têm demonstrado a grande relevância de características socioeconômicas e demográficas como fatores condicionantes à inserção no mercado de trabalho. Além disso, Barros et al. (1997) chamam atenção para o fato de ser o desemprego ameaça constante no cenário econômico da década de 1990 e, somando-se a isso, a qualidade dos postos de trabalho tem se destacado como preocupação persistente, sendo esse fator significativo na promoção da desigualdade e pobreza.

Corseuil et al. (1997) enfatizaram a forte tendência observada pela variáveis sexo, ida- 
de, escolaridade e posição no domicílio como fatores que variam na composição do desemprego metropolitano brasileiro sem, contudo, destoar acentuadamente nos resultados encontrados. Diante disso, é clássica a hipótese de que variáveis como sexo, idade, raça/cor têm forte influência para determinar o desemprego no Brasil.

Barros (2010) observou a forte relação entre as variáveis supracitadas na determinação do desemprego metropolitano brasileiro. Com isso, as evidências empíricas têm comprovado que características socioeconômicas e demográficas são determinantes tanto para estabelecer a relação causa e efeito no desemprego quanto para observar a permanência dessa PEA no desemprego brasileiro.

Além disso, as características regionais têm forte influência tanto no perfil do desemprego quanto na duração (CORSEUIL et al., 1999). Concomitante a isso, Silva Filho (2011) observou a desigualdade nas características do desempregado nas regiões metropolitanas do nordeste, sendo constatado que as mulheres, os não brancos, os jovens e os idosos sofrem mais com o desemprego nos anos 2000.

Diante disso, a seção que segue busca tratar dos procedimentos metodológicos de forma a permitir observar o desemprego no estado do Rio Grande do Norte e na região metropolitana de Natal nos anos selecionados.

\section{PROCEDIMENTOS METODOLÓGICOS}

FIGURA 1 - RIO GRANDE DO NORTE E A REGIÃO METROPOLITANA DE NATAL

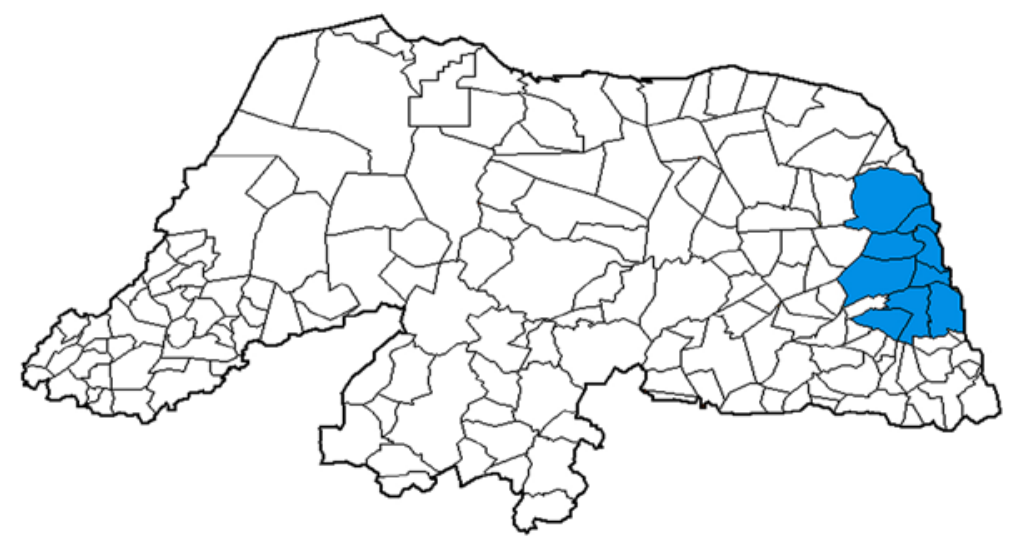

FONTE: Mapa adaptado a partir do Atlas do Desenvolvimento Humano da PNUD.

Para o alcance dos objetivos propostos pelo estudo, pretende-se fazer uma análise da participação relativa e dos determinantes do desemprego: inicialmente, tabularam-se os dados a partir dos microdados da Pesquisa Nacional por Amostra de Domicílios (PNAD), na série temporal que compreende os anos de 2001 a 2008. Posteriormente, construíram-se os indica- 
dores de composição do desemprego para o estado do Rio Grande do Norte e para a região metropolitana de Natal.

Para a construção da composição do desemprego, utilizaram-se variáveis como:

- Estoque total de desempregados;

- Estoque de desempregados segundo o sexo;

- Estoque de desempregados segundo a raça/cor;

- Estoque de desempregados segundo a faixa etária;

- Estoque de desempregados segundo os anos de estudo.

A metodologia aqui aplicada para obtenção dos resultados foi utilizada inicialmente por Corseuil et al. (1997) com dados da Pesquisa Mensal do Emprego (IBGE) para as metrópoles brasileiras, no período que compreende os anos de 1986 e 1995 e, posteriormente, por Bastos (2010) com dados da Pesquisa de Emprego e Desemprego para as metrópoles brasileiras e para o Distrito Federal, na série temporal entre 1999 e 2007. Replicando a metodologia dos autores para o estudo no estado do Rio Grande do Norte e na região metropolitana de Natal, o desemprego de um grupo populacional de uma região pode ter a seguinte decomposição:

$$
U i / U=U i / N i . N i / P i . P i / P . P / N . N / U
$$

Onde $U i=$ caráter de estoque de desempregados do grupo $i$ no estado do $\mathrm{RN}$ e na RMN; $U=$ estoque de desempregados total no estado do RN e na RMN nos anos selecionados; $N i=$ número de membros da PEA do grupo $i$ tanto no estado quanto na RM; $N=$ número de membros da PEA total no estado do RN e na RMN nos anos selecionados; $P i=$ número de membros da PIA do grupo $i$; $P=$ número de membros da PIA total no estado do RN e na RMN no período em análise.

Essa denominação permite reescrever a representação da forma que se segue:

$$
U i / U=\frac{U i / N i}{U / N} \cdot \frac{N i / P i}{N / P} \cdot \frac{P i}{P}
$$

Da expressão anterior, transformam-se os dados da Equação (2) em logaritmos naturais, uma vez que o objetivo aqui é captar o efeito elasticidade na decomposição dos fatores que 
afetam o desemprego.

Dessa forma, tem-se:

$$
\operatorname{Ln}(U i / U)=[\operatorname{Ln}(U i / N i)-\operatorname{Ln}(U / N)]+[\operatorname{Ln}(N i / P i)-\operatorname{Ln}(N / P)]+\operatorname{Ln}(P i / P)
$$

Com esta expressão, têm-se as variações expressas da seguinte forma:

$$
\Delta \operatorname{Ln}(U i / U)=[\Delta \operatorname{Ln}(U i / N i)-\Delta \operatorname{Ln}(U / N)]+[\Delta \operatorname{Ln}(N i / P i)-\Delta \operatorname{Ln}(N / P)]+\Delta \operatorname{Ln}(P i / P)
$$

Apresentado o método, a expressão (4) propõe observar a variação da participação do grupo $i$ no estoque de desempregados de uma região. Interpretando Corseuil et al. (1997) e Bastos (2010), a participação dos desempregados de um determinado grupo $i$ em dada região está diretamente relacionada à variação do peso deste grupo populacional na PIA da região; a taxa de participação da força de trabalho do grupo $i$ à taxa de participação da força de trabalho da região; e a participação da taxa de desempregos $i$ à taxa total de desempregados existentes na região.

\section{DISCUSSÃO DOS RESULTADOS}

Os resultados aqui encontrados mostram que todos os fatores contribuíram para a redução da taxa de desemprego masculino no estado do $\mathrm{RN}$ no confronto entre os anos de 2001 e 2008. Além disso, na RMN, com exceção da variação da taxa de participação dessa força de trabalho na força de trabalho total da região, todas as demais variáveis também contribuíram para retirar a força de trabalho masculina do desemprego metropolitano da RMN.

TABELA 1 - EVOLUÇÃO DA PARTICIPAÇÃO SEGUNDO O SEXO NO DESEMPREGO E SEUS COM-

\begin{tabular}{|c|c|c|c|c|}
\hline \multirow{2}{*}{ Discriminação } & \multicolumn{2}{|c|}{ Masculino } & \multicolumn{2}{|c|}{ Feminino } \\
\hline & $\mathbf{R N}$ & $\mathbf{R M N}$ & $\mathbf{R N}$ & $\mathbf{R M N}$ \\
\hline$\Delta \ln (\mathrm{Ui} / \mathrm{U})$ & & & & 0,228 \\
\hline$\Delta \ln (\mathrm{Ui} / \mathrm{Ni})-\Delta \ln (\mathrm{U} / \mathrm{N})$ & & & & 0,216 \\
\hline$\Delta \ln (\mathrm{Ni} / \mathrm{Pi})-\Delta \ln (\mathrm{N} / \mathrm{P})$ & & & & 0,010 \\
\hline$\Delta \ln (\mathrm{Pi} / \mathrm{P})$ & & & & 0,003 \\
\hline
\end{tabular}
PONENTES, ENTRE 2001 E 2008 - RN E RMN FONTE: PNAD, 2001-2008/IBGE.

Porém, quando observada a composição do desemprego feminino, constata-se que todos os fatores contribuíram para elevar a participação da força de trabalho das mulheres no desemprego tanto no estado do RN quanto na RMN. Para tanto, o fator que mais corroborou à elevação da força de trabalho feminina no desemprego foi a redução da taxa de desemprego 
feminina em comparação à taxa de desemprego na região, tendo essa registrado 0,226 no estado do RN e 0,216 na RMN.

Esses resultados, portanto, corroboram a literatura, ratificando maior vulnerabilidade ao desemprego para a força de trabalho feminina, superior à força de trabalho masculina. Tais evidências foram constatadas tanto em todo o estado do RN quanto na RMN.

Quando se observa a variável raça/cor, as evidências empíricas encontradas no RN e na $\mathrm{RMN}$ ratificam a literatura, haja vista que no estado do $\mathrm{RN}$ todos os fatores contribuíram para a retirada da população de raça/cor branca do desemprego, assim como na RMN, com exceção da variação da força de trabalho em relação à força de trabalho total da região, para a RM. Esses resultados mostram que nos anos em análise houve uma forte participação da composição do desemprego aqui analisada para reduzir o desemprego da população branca no estado e na região metropolitana.

TABELA 2 - EVOLUÇÃO DA PARTICIPAÇÃO SEGUNDO A RAÇA/COR NO DESEMPREGO E SEUS COMPONENTES, ENTRE 2001 E 2008 - RN E RMN

\begin{tabular}{l|rrr|r}
\hline \multicolumn{1}{c|}{ Discriminação } & \multicolumn{2}{c|}{ Branco } & \multicolumn{2}{c}{ Não branco } \\
\cline { 2 - 5 } & RN & RMN & \multicolumn{1}{c}{ RN } & \multicolumn{1}{c}{ RMN } \\
\hline$\Delta \ln (\mathrm{Ui} / \mathrm{U})$ & $-0,135$ & $-0,432$ & 0,025 & 0,358 \\
$\Delta \ln (\mathrm{Ui} / \mathrm{Ni})-\Delta \ln (\mathrm{U} / \mathrm{N})$ & $-0,062$ & $-0,189$ & $-0,015$ & 0,168 \\
$\Delta \ln (\mathrm{Ni} / \mathrm{Pi})-\Delta \ln (\mathrm{N} / \mathrm{P})$ & $-0,006$ & 0,005 & 0,000 & $-0,013$ \\
$\Delta \ln (\mathrm{Pi} / \mathrm{P})$ & $-0,066$ & $-0,248$ & 0,039 & 0,203 \\
\hline
\end{tabular}

FONTE: PNAD, 2001-2008/IBGE.

Contrariamente ao acima exposto, somente o fator variação do peso do grupo de não brancos na PIA da região contribuiu para retirar esses do desemprego no estado do RN. Já na RMN, apenas a redução da taxa de participação da força de trabalho de não brancos na taxa de participação da força de trabalho da região mostrou-se corroborar. Assim, percebe-se a forte contribuição das demais variáveis para elevar a taxa de participação dos não brancos no desemprego total tanto no estado do RN quanto na RMN.

Quanto a idade da PEA, os resultados observados mostram destoar entre as faixas etárias a contribuição de cada uma no desemprego do RN e da RMN. Para a faixa etária entre 10 e 14 anos, constata-se forte oscilação entre os anos observados: tanto a variação do peso da PIA do grupo na PIA total quanto a taxa de participação da força de trabalho na taxa de participação da força de trabalho na região contribuíram para retirar essa PEA do desemprego no estado do RN. No entanto, a variação do peso desse grupo populacional na PIA dessa região contribuiu fortemente para levá-los ao desemprego. Na RMN, somente a variação do peso dessa PIA na PIA da região contribuiu para retirá-los do desemprego; os demais fatores variaram em sentido contrário. 
$\mathrm{Na}$ faixa etária entre 15 e 24 anos, somente o comportamento da taxa de desemprego do grupo em relação à taxa de desemprego da do estado não comprovou à retirada dessa PEA do desemprego no estado do RN. Na RMN, somente a variação do peso da PIA em relação ao peso da PIA da região teve participação para retirá-los do desemprego $(-0,213)$. Já na faixa etária entre 25 e 39 anos, esse fator foi o único que não contribuiu para retirar essa PEA do desemprego. No estado do RN, nessa mesma faixa etária, todos os fatores, com exceção do comportamento da força de trabalho do grupo em relação à força de trabalho da região, direcionaram essa PEA ao desemprego.

TABELA 3 - EVOLUÇÃO DA PARTICIPAÇÃO SEGUNDO A FAIXA ETÁRIA NO DESEMPREGO E SEUS COMPONENTES, ENTRE 2001 E 2008 - RN E RMN

\begin{tabular}{|c|c|c|c|c|c|c|c|c|c|c|}
\hline \multirow[t]{2}{*}{ Discriminação } & \multicolumn{2}{|c|}{ De 10 a 14} & \multicolumn{2}{|c|}{ De 15 a 24} & \multicolumn{2}{|c|}{ De 25 a 39} & \multicolumn{2}{|c|}{ De 40 a 59} & \multicolumn{2}{|c|}{$\begin{array}{c}60 \text { anos ou } \\
\text { mais }\end{array}$} \\
\hline & $\mathbf{R N}$ & RMN & $\mathbf{R N}$ & RMN & $\mathbf{R N}$ & RMN & $\mathbf{R N}$ & RMN & RN & RMN \\
\hline$\overline{\Delta \ln (\mathrm{Ui} / \mathrm{U})}$ & 1,384 & 0,786 & $-0,239$ & $\begin{array}{c}-0,104 \\
\end{array}$ & 0,179 & $-0,082$ & $-0,047$ & 0,197 & 0,471 & 0,056 \\
\hline$\Delta \ln (\mathrm{Ui} / \mathrm{Ni})-\Delta \ln (\mathrm{U} / \mathrm{N})$ & 1,659 & 0,320 & $-0,056$ & 0,082 & 0,186 & $-0,079$ & $-0,201$ & 0,063 & 0,249 & $-0,228$ \\
\hline$\Delta \ln (\mathrm{Ni} / \mathrm{Pi})-\Delta \ln (\mathrm{N} / \mathrm{P})$ & $-0,180$ & 0,562 & 0,030 & 0,027 & $-0,036$ & $-0,031$ & $-0,016$ & $-0,036$ & 0,078 & 0,141 \\
\hline$\Delta \ln (\mathrm{Pi} / \mathrm{P})$ & $-0,095$ & $-0,095$ & $-0,213$ & $-0,213$ & 0,029 & 0,029 & 0,170 & 0,170 & 0,143 & 0,143 \\
\hline
\end{tabular}

FONTE: PNAD, 2001-2008/IBGE.

Para a faixa etária entre 40 e 59 anos, somente a variação do peso do grupo na PIA da região não confirmou à retirada dessa PEA do desemprego; os demais fatores comportaram-se de forma a retirá-los do desemprego em todo o estado. Já na RMN, o único fator que contribuiu para retirá-los do desemprego foi o comportamento da taxa de participação da força de trabalho do grupo em comparação com a força de trabalho da região. Na faixa etária acima de 60 anos, todos os fatores contribuíram para levá-los ao desemprego no estado do RN, sendo que na RMN o comportamento da taxa de desemprego do grupo em relação à taxa de desemprego da região contribuiu positivamente para retirar essa PEA do desemprego. Esse resultado, portanto, ratifica a hipótese de que os idosos são mais vulneráveis ao desemprego no Brasil.

Quanto aos anos de estudo da PEA, para aqueles com escolaridade entre 0 e 4 anos, todos os fatores contribuíram para retirá-los do desemprego no estado do RN. Na RMN, com exceção do comportamento da taxa de desemprego do grupo, em relação ao desemprego da região, os demais fatores seguiram a tendência observada no estado. Com isso, observa-se que a PEA com a menor escolaridade obteve menores índices no desemprego no RN e na RMN, haja vista que esse grupo está mais vulnerável às várias formas de trabalho e aceita com mais facilidade qualquer tipo de emprego.

Para a PEA com escolaridade entre 5 e 8 anos de estudo, com exceção do comportamento da taxa de desemprego do grupo em comparação com a taxa de desemprego da região, 
as demais variavéis contribuíram para retirar eles do desemprego no estado do RN. Já na RMN, somente a variação do peso do grupo na PIA total da região variou de forma a retirá-los do desemprego.

TABELA 4 - EVOLUÇÃO DA PARTICIPAÇÃO SEGUNDO ANOS DE ESTUDO NO DESEMPREGO E SEUS COMPONENTES, ENTRE 2001 E 2008 - RN E RMN

\begin{tabular}{|c|c|c|c|c|c|c|c|c|}
\hline \multirow{2}{*}{ Discriminação } & \multicolumn{2}{|c|}{ De 0 a 4} & \multicolumn{2}{|c|}{ De 5 a 8} & \multicolumn{2}{|c|}{ De 9 a 11} & \multicolumn{2}{|c|}{12 para cima } \\
\hline & $\mathbf{R N}$ & RMN & $\mathbf{R N}$ & $\mathbf{R M N}$ & $\mathbf{R N}$ & RMN & $\mathbf{R N}$ & RMN \\
\hline$\overline{\Delta \ln (\mathrm{Ui} / \mathrm{U})}$ & $-0,808$ & $-0,220$ & $-0,037$ & 0,431 & 0,691 & 0,621 & 1,684 & 1,459 \\
\hline$\Delta \ln (\mathrm{Ui} / \mathrm{Ni})-\Delta \ln (\mathrm{U} / \mathrm{N})$ & $-0,573$ & 0,134 & 0,062 & 0,627 & $-0,110$ & 0,591 & 1,343 & 1,234 \\
\hline$\Delta \ln (\mathrm{Ni} / \mathrm{Pi})-\Delta \ln (\mathrm{N} / \mathrm{P})$ & $-0,058$ & $-0,201$ & $-0,018$ & 0,014 & 0,455 & 0,408 & $-0,113$ & $-0,054$ \\
\hline$\Delta \ln (\mathrm{Pi} / \mathrm{P})$ & $-0,177$ & $-0,153$ & $-0,081$ & $-0,211$ & 0,346 & 0,220 & 0,454 & 0,279 \\
\hline
\end{tabular}

FONTE: PNAD, 2001-2008/IBGE.

$\mathrm{Na}$ faixa de 9 a 11 anos de estudo, somente o comportamento da taxa de desemprego do grupo em relação ao desemprego da região variou de forma a retirar essa PEA no estado do RN do desemprego. Além disso, na RMN, todos os fatores contribuíram para levá-los ao desemprego nos anos observados. Já para a faixa de escolaridade acima de 12 anos de estudo, somente o comportamento da taxa de participação da força de trabalho do grupo em comparação com a força de trabalho da região contribuiu positivamente para retirar essa PEA do desemprego, tanto no estado do RN (-0,113) quanto na RMN $(-0,054)$.

\section{CONSIDERAÇÕES FINAIS}

O objetivo deste artigo foi fazer uma avaliação do desemprego no estado do Rio Grande do Norte e a na região metropolitana de Natal nos anos entre 2001 e 2008. Os resultados encontrados mostram redução do desemprego na RMN a partir de 2003, diferentemente do observado para todo o estado, que só apresenta redução contínua a partir de 2005.

Além disso, o desemprego no estado e na RMN tem afetado, sobremaneira, as mulheres e os não brancos, seguindo a tendência observada nas regiões metropolitanas brasileiras e em todo o país. Tanto no RN quanto na RMN o desemprego feminino foi superior ao masculino em todos os anos analisados. Já em relação à raça/cor, somente no ano de 2007, e no estado do RN, a taxa de desemprego para não brancos foi inferior a observada para os brancos. Tais resultados refletem a discriminação no mercado de trabalho já amplamente difundida na literatura.

Quanto à faixa etária mais atingida pelo desemprego, podem-se destacar aqueles com idade entre 15 e 24 anos e 25 e 39, tanto no estado quanto na região metropolitana. Além disso, merece atenção as menores taxas de desemprego observadas para a população com idade acima 
de 60 anos, seja pela necessidade de complemento da renda familiar, seja pela necessidade de manterem-se no mercado do trabalho, mesmo sem escolhas de melhores empregos; esse foi o grupo menos afetado pelo desemprego no estado do RN e na RMN.

Em relação à escolaridade da PEA, constatou-se que as faixas de 0 a 4 anos de estudo e acima de 12 anos foram as menos atingidas pelo desemprego, tanto no RN quanto na RMN. Considere-se que, para a primeira faixa, há mais possibilidades de trabalhos em setores que não demandam melhores níveis de estudos e, dessa forma, são maiores as possibilidades de trabalhos, mesmo se sabendo das condições precárias que lhes são impostas. Para os últimos, existem melhores possibilidades de trabalho, dada a melhor formação dessa PEA.

O estudo, portanto, evidenciou alguns determinantes do desemprego e o perfil do desemprego no estado do RN e na RMN, sendo o desemprego mais acentuado para a RM, além de divergências observadas em relação aos determinantes do desemprego. Observaram-se características inerentes ao mercado de trabalho nacional, assim como aqueles que são peculiares de regiões com dinâmica econômica menos acentuada. No geral, percebeu-se um mercado de trabalho seletista e excludente, bem como relativa redução nas taxas de desemprego do estado e da RMN.

\section{REFERÊNCIAS}

ANTUNES, R. Adeus ao trabalho? Ensaios sobre as metamorfoses e a centralidade do mundo do trabalho. 10 ed. São Paulo: Editora da Universidade Estadual de Campinas, 2005.

BALTAR, P. E. A. Estrutura econômica e emprego urbano na década de 1990. In: PRONI, M. W.; HENRIQUE, W. (Org.). O Brasil nos anos 90. São Paulo: UNESP; Campinas: UNICAMP, 2003.

BARROS, R. P.; CAMARGO, J. M. ; MENDONÇA, R. A estrutura do desemprego o Brasil. IPEA, n. 487, 1999. Discussão.

BASTOS, R. L. A. Desemprego metropolitano no Brasil. In: ENCONTRO NACIONAL DA ASSOCIAÇÃO BRASILEIRA DE ESTUDOS POPULACIONAIS. Anais... Caxambú: ABEP, 2010.

CARVALHO, I. M. M. de. Globalização, metrópoles e crise social no Brasil. Revista EURE, Santiago de Chile, v. 32, n. 95, p. 5-20, mai. 2006.

CLEMENTINO, M. L. M.; SILVA, M. G. da; PEREIRA, W. E. N. Transformações recentes na economia da região metropolitana de Natal. In: CLEMENTINO, M. L. M.; PESSOA, Z. S. (Org.). Natal: Uma metrópole em formação. São Paulo: EDUC: PUC-SP, 2009.

CORSEUIL, C. et al. Determinantes da evolução da estrutura do desemprego no Brasil: 19861995. Revista de Economia Aplicada, São Paulo, v. 1, n. 3, p. 443-467, 1997.

CORSEUIL, C. et al. Desemprego regional no Brasil: uma abordagem empírica. Revista de Economia Aplicada, v. 3, n. 3, 1999. 
DEDECCA, C. S.; ROSANDISKI, E. N. Recuperação econômica e geração de empregos formais. Revista Parcerias Estratégicas, Brasília, n. 22, jun. 2006.

HIRATA, H. A precarização e a divisão internacional e sexual do trabalho. Revista Sociologias, Porto Alegre, ano 11, n. 21, p. 24-41, jan./jun. 2009.

NEVES, M. A.; PEDROSA, C. M. Gênero, flexibilidade e precarização: o trabalho a domicilio na indústria de confecções. Revista Sociedade e Estado, Brasília, v. 22, n. 1, p. 11-34, jan./ abr., 2007.

POCHMANN, M. O trabalho sob fogo cruzado: exclusão, desemprego e precarização no final do século. São Paulo: Contexto, 1999.

REMY, M. A. P. A.; QUEIROZ, S. N.; SILVA FILHO, L. A. Evolução recente do emprego formal no Brasil - 2000-2008. In: ENCONTRO NACIONAL DA ASSOCIAÇÃO BRASILEIRA DE ESTUDOS POPULACIONAIS, 17., 2010, Caxambú. Anais... Caxambú: ABEP, 2010.

SILVA FILHO, L. A.; QUEIROZ, S. N. A trajetória da indústria e do emprego formal no Ceará - 1996/2006. In: ENCONTRO NACIONAL DE ESTUDOS DO TRABALHO, 11., 2009, Campinas. Anais... Campinas: ABET, 2009.

SILVA FILHO, L. A. Mercado de trabalho e estrutura sócio-ocupacional: estudo comparativo entre as regiões metropolitanas de Fortaleza, Recife e Salvador - 2001-2008. Dissertação (Mestrado em Economia) - Universidade Federal do Rio Grande do Norte, Natal, 2011. 
